"Schneider" — 2009/2/18 — 23:40 — page 379 — \#1

\title{
CAS as a didactical challenge
}

\author{
EDITH SCHNEIDER
}

Abstract. The paper starts with the discussion of a concept of general mathematics education (mathematics education for everyone). This concept views the focus of teaching mathematics in the reduction of the demands in the field of operative knowledge and skills as well as in an increase of the demands in the fields of basic knowledge and reflection. The consequences of this concept are didactically challenging for the use of Computer Algebra Systems (CAS) in the teaching of mathematics. By reducing the operative work we reduce exactly that field in which the original potential of CAS lies. It is shown that in such maths classes the main focus of CAS is on their use as a pedagogical tool, namely as support for the development of basic knowledge and reflection as well as a model of communication with mathematical experts.

Key words and phrases: general mathematics education, Computer Algebra Systems, CAS-supported mathematics teaching, basic knowledge, reflection, reduction of operative knowledge, communication with mathematical experts, pedagogical tool.

ZDM Subject Classification: B10, D30, D40, R20, U50.

\section{Introduction}

Let us start with the question: what is specific for CAS?

Computer Algebra Systems (CAS) can operate by mathematical rules on mathematical objects represented by symbols. That means, CAS can master a lot of numerical and algebraic operations and manipulations - in particular most of the numerical and algebraic operations and manipulations dealt with in school. CAS were originally developed exactly with this aim and it is this capability of CAS that makes up the specific strength and potential of CAS.

Copyright (C) 2008 by University of Debrecen 


$$
\text { "Schneider" — 2009/2/18 — 23:40 — page 380 — \#2 }
$$

In this possibility of CAS a "danger" for the mathematics lessons can be seen: operating on symbols using rules is an essential component of mathematics as well as a characteristic feature of mathematics. As a mathematician as well as a professional user of mathematics one needs operative knowledge and skills; in particular one should know how to use the operative possibilities in a creative way - to generate new mathematical knowledge/theories, to solve ("real-world") problems. Thus, to learn mathematics means to learn (at least) first steps of what mathematicians are doing and how they are doing it - mathematics education means to produce mini-mathematicians (mathematical "mini-experts"). Such a position will see operational work as an essential part of the mathematical education and it will require that this knowledge and these skills are practiced and trained in the maths lessons. Firstly, one needs to know and master the various numerical and algebraic operations by oneself. If at all, only after mastering this, CAS can be used. This position of educating "mini-experts" is neither sensible nor practicable. One can become an expert or be an expert only in a very limited number of fields.

Another reaction to the possibility of CAS is that by using CAS the development of operative abilities and skills could be abandoned in the mathematics lessons. Operative work could be outsourced to CAS, and hence didactically more relevant contents and activities could be dealt with. This second position closely follows the demands of many mathematics educators for reducing the operative work in the teaching of mathematics (e.g., [12], [13]). It does not focus on "producing" mathematical mini-experts but rather on mathematical education for everyone (general mathematics education). Such positions are usually based on theories referring to educational considerations (the actual and future role of the individual within society and the role of the specific field, in our case mathematics, for society).

There exist different educational theories for mathematics education. One of these is the concept of higher general mathematics education of Roland Fischer. This concept is the base of our considerations concerning the use of CAS in teaching mathematics (see e.g. [5], [6], [7], [8], [9], [10]). I will briefly introduce this concept before discussing the role of CAS for mathematics education. 


$$
\text { "Schneider" — 2009/2/18 — 23:40 — page 381 — \#3 }
$$

\section{R. Fischer's concept of (higher) general mathematics education}

In his concept of (higher $)^{1}$ general mathematics education Fischer starts from a thesis based on socio-philosophical aspects: the functionality of our highly differentiated democratic society, built up on the distribution of labor, is essentially based on emancipated contact with highly specialized expert knowledge. As mature, responsible citizens we are constantly confronted with statements made by experts, which we must then assess and judge in order to be able to make (our own) decisions - without being experts in the field ourselves.

Hence, the main aim of (higher) education should be the ability to handle expert knowledge in an emancipated and critical way: that is to communicate with experts in an emancipated and reflected way. Those persons who have attended institutes like high schools and vocational high schools ("the more highly educated", "the generally educated") should particularly be able to ask the experts the "right" questions as well as to understand and to explain the experts' statements in an understandable way. And they should be able to judge the importance of the experts' statements and to make (their own) decision (cf. [3], [4]).

Therefore, other competences are necessary than those one needs to be or to become an expert. Fischer identifies the following three fields of competence as those which can be acquired for every subject: basic knowledge (basic notions, concepts, forms of representation), operative knowledge and skills (problem solving, proofs, in general: generating/creating new knowledge), and reflection (possibilities, limits and meaning of concepts and methods).

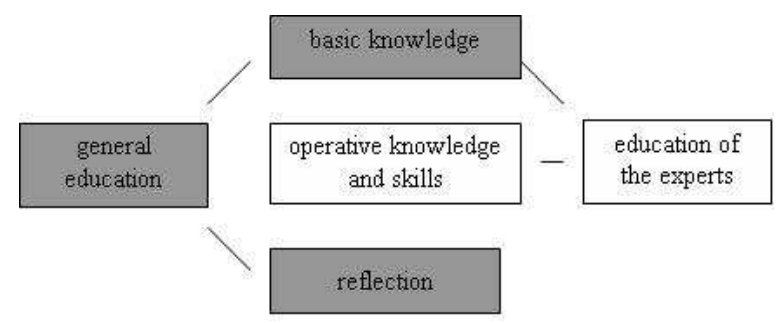

Figure 1. Fields of competence (acc. to [3], [4, p. 5])

Experts should be competent particularly in the first two fields (with main focus on the field of operative knowledge and skills because of its relevance for

${ }^{1}$ The concept mainly focuses on mathematics education of grade $9-12 / 13$.
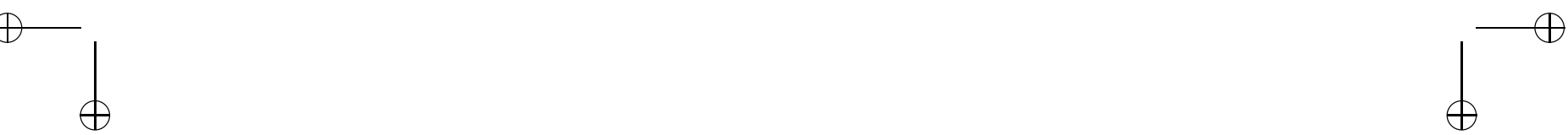
problem solving as well as for generating/creating new knowledge). For the generally educated layperson Fischer considers the fields of basic knowledge and reflection to be particularly important (cf. Figure 1). He justifies it with regard to the ability to communicate with experts: basic knowledge "is a prerequisite for communicating with experts", reflection "is necessary for judging their expertise" $([4$, p. 5$])$.

Fischer points out that his classification should not be taken as an absolute and that it does not mean that doing operations should be completely removed from a (general) mathematics education. However, the focus and profiles of competences for experts and laypersons clearly differ.

The consequence for teaching of mathematics for everyone is, in short ([4, p. 6]) to reduce the demands in the field of operative knowledge and skills and to increase the demands in the fields of basic knowledge and reflection.

It is interesting that National Standards for School Mathematics - at least in Austria and Germany (cf. [1], [11]) - have a quite similar basic orientation and also the demands of the 84 PISA-Mathematics test-items focus more on the fields of basic knowledge and reflection than on the field of operative knowledge and skills.

This orientation often differs from the present practice of mathematics teaching. To some extent it requires strong modifications concerning the contents of mathematics teaching and the tasks treated in mathematics classrooms (cf. Figure $2(\mathrm{a}), 2(\mathrm{~b}))$.

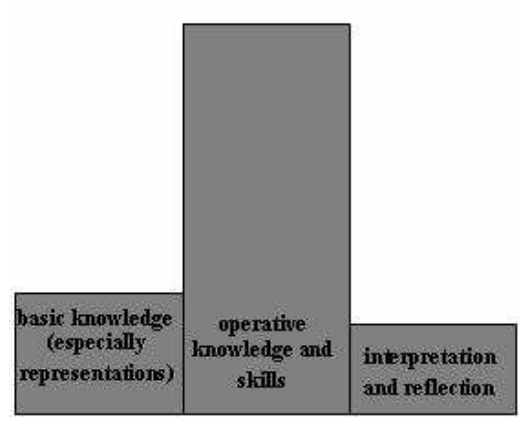

(a) Traditional teaching of mathematics

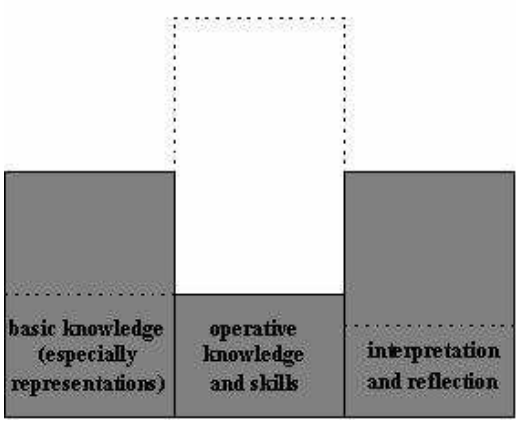

(b) Teaching in the sense of general mathematics education

Figure 2
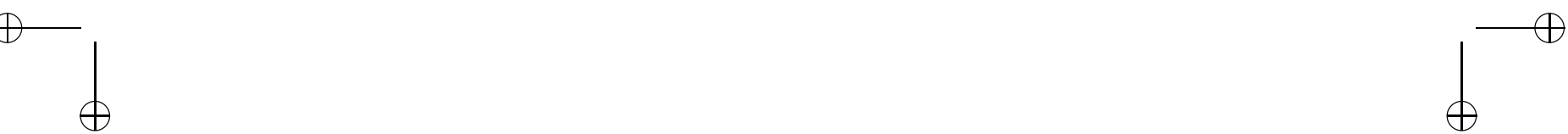


$$
\text { "Schneider" — 2009/2/18 — 23:40 — page 383 — \#5 }
$$

Following Fischer's concept of mathematics education all operational work requiring more than elementary transformations is primarily experts' knowledge. One can give up operative work in the math classroom to a large extent. The consequence is that mathematics teaching focusing on such a general mathematics education has limited contents where the original potential of CAS (namely algebraic operations) really comes in useful. (In a doctoral thesis at our institute which focused on the design of curricula following Fischer's concept we were able to observe exactly this effect.) This rouses the question, whether or not one needs CAS (or in general: computers) in mathematics classrooms any longer.

We see at least two essential aspects where CAS can make relevant contributions in supporting mathematics teaching focusing on mathematics education for everyone:

1. CAS as support for the development of basic knowledge and reflection

2. Using CAS as a model for communication with experts

\section{Basic knowledge and reflection with CAS}

By CAS operational knowledge and skills can be used by the students without the need of being developed by the students themselves. Thus, experts' knowledge is available for the students in the maths classroom to a large extent. This feature of CAS can be used for the development of basic knowledge and reflection. In this case, four fields seem to be most relevant:

- representation

- exploration

- elementarisation

- modularisation

\subsection{Representation}

With regard to the development of basic knowledge and reflection, the main aspect seems to be that CAS enables a rapid and flexible availability of different symbolic, graphical and tabular representations. This easy availability of CAS-representations now offers the possibility to use various representations quite frequently. Thus, students can get to know basic representations of mathematics much better (which Fischer explicitly assigns to the field of basic knowledge); the 
students have more opportunities to learn to interpret the different representations, to use them, and to communicate through them and about them. With regard to reflection, they can experience and think about the purpose of the various forms of representation as well as about their advantages and disadvantages.

Additionally, an interesting aspect is that CAS-representations do not only replace representations constructed by hand, they can also lead to a shift in their meanings. One example are graphical representations of functions and their meaning and significance for the investigation of the behaviour and the characteristics of functions with and without the availability of CAS.

\subsection{Exploration}

CAS make explorative working for the mathematics classrooms accessible by taking over the operative work.

An explorative approach can be used for the development of basic knowledge as well as for interpretations and reflection of essential (basic) characteristics of mathematical concepts. Here, an interesting aspect lies on the possibility to investigate and to identify (in an explorative way) such important characteristics or features of mathematical notions on one's own. Such an example could be an explorative investigation of the relation between different parameters of a function and the behaviour of the function (i.e. Figure 3), an exploration of the characteristics of the differentiability or non-differentiability of a function (i.e. Figure 4), looking for the relationship between lower sums, upper sums, and integral, etc.

The investigations are often carried out in an interplay between symbolic and schematic (especially graphical) representations. Starting points can be the symbolic as well as the schematic representation.

In addition, explorative working supports competences that are (also) relevant for the communication between (mathematical) experts and (generally educated) laypersons. Such competences are, for instance, to ask CAS "appropriate" questions, to present the questions in a CAS-compatible way, to interpret the CAS-results/outputs in a mathematically and referentially correct way, to classify and to judge the results.

\subsection{Elementarisation}

One of the fundamental aims of mathematics is to create general "closed" representations for (different) problems. Examples are the "closed" formulas for 


$$
\text { "Schneider" — 2009/2/18 — 23:40 — page 385 — \#7 }
$$
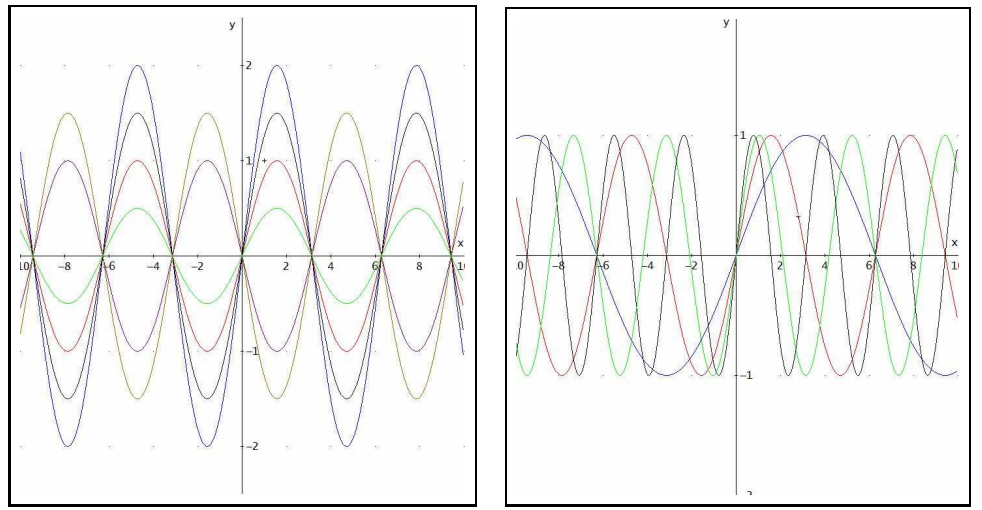

Figure 3. sine function $f: f(t)=a \cdot \sin (\omega \cdot t+\rho)$ - variation of parameter $a$ (amplitude) or $\omega$ (frequency) with $\rho=0$

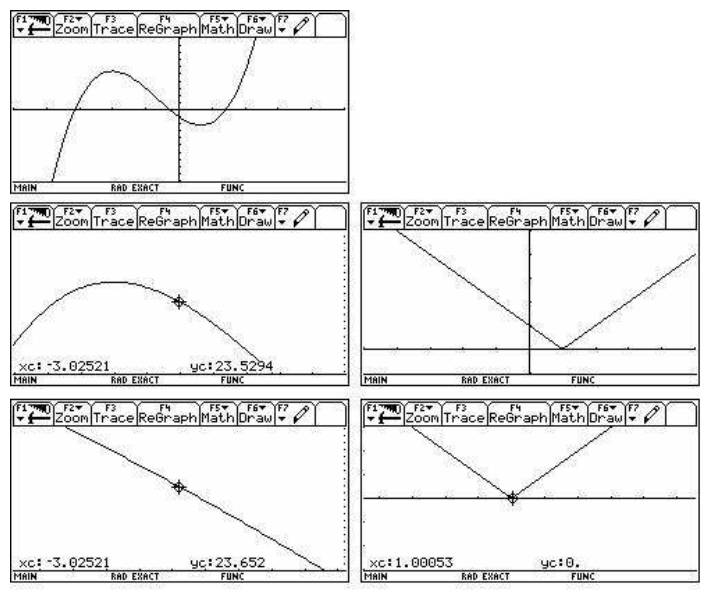

Figure 4

solving algebraic equations (such as quadratic equations), closed formulas for derivatives and antiderivatives of real functions, etc.

One intention of looking for such closed representations is that such compact mathematical formulas and methods have the advantage that they considerably reduce the operative work for determining solutions. For example, by means of the formula for the final value of an annuity $E_{n}=R \cdot \frac{(1+i)^{n}-1}{i}$ one can determine rapidly the final values for different rates $R$, interests $i$, and periods $n$. However, 


$$
\text { "Schneider" — 2009/2/18 — 23:40 — page 386 — \#8 }
$$

at the same time the compact representation of closed formulas reduces their transparency. The constitutive elements of mathematical concepts can often not be seen explicitly in the compact and closed representations. So, the formula for the final value of an annuity hardly gives explicit information about its basic components. (Which model of payment of interest is used? How to come to the final value of the next year?)

A more elementary method for calculating the same final value of an annuity is:

$$
\begin{aligned}
& E_{1}=R \\
& E_{2}=E_{1} \cdot(1+i)+R \\
& E_{3}=E_{2} \cdot(1+i)+R \\
& \quad \vdots \\
& E_{n}=E_{n-1} \cdot(1+i)+R
\end{aligned}
$$

This elementary method is highly transparent and comprehensible. It is directly clear and comprehensible in which way the rates and interests are added and consequently which model of payment of interest is used. The disadvantage of this procedure is as evident: the determination of the final value of a monthly paid rate requires 180 lines with many calculations; such a procedure is hardly reasonable when it is necessary to perform it step by step (even when using a simple (pocket-)calculator).

When using CAS elementary representations and methods become available for mathematics teaching because of the possibility of outsourcing the operative work to CAS. By using elementary procedures to make the constitutive characteristics of mathematical concepts and procedures directly clear and comprehensible the development of reflected basic knowledge could be supported. One well known and convincing example for such an approach is the use of recurrent representations. For instance, these representations have a high quality of explanation when investigating linear or exponential functions and their constitutive characteristics of constant (absolute or relative) rates of change. A recurrent representation expresses this characteristic more clearly than other symbolic representations: by means of the recurrent equation of the linear function $N(t)=N(t-1)+a$ or the exponential function $N(t)=N(t-1)+r \cdot N(t-1)($ or $N(t)=a \cdot N(t-1))$ the constantly absolute or relative increase per (time) unit is directly recognizable/"visible". Figure 5 shows the recurrent representation of an exponential growth used for determining concrete values. 


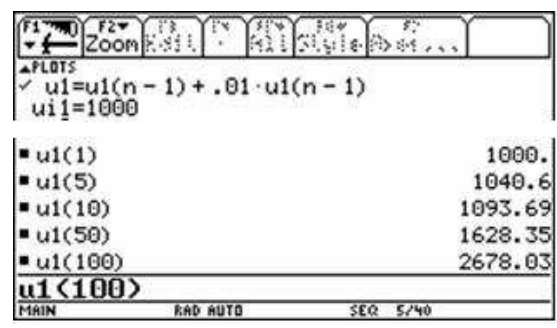

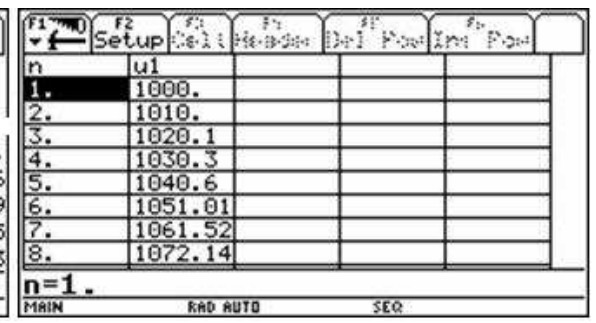

Figure 5

The "closed" algebraic representation of the linear function $f(x)=a x+b$ or the exponential function $f(x)=c a^{x}$ requires additional knowledge to recognize the constitutive characteristic within it. This characteristic of linear or exponential growth also cannot be directly recognized in or from the graph of the function. When using tables it is necessary to compare the rates of change and to identify the constant value (typical at least for the limited range).

\subsection{Modularisation}

A module will be seen here as a compressed unit of knowledge which can be used as a whole without necessarily being explicitly unfoldable into its components, i.e. it is not necessary to be able to "look inside" the module (cf. [2]).

The bundling of linked information to units (modules) which need not be unfolded when using them could structure complex thinking processes and actions and reduce their complexity. In such a way, modularisation could considerably facilitate our thinking and acting.

When working with CAS we are dealing with modules which are the same ones present without CAS: a module for division, a module for multiplication, a module for solving quadratic equations, a module for plotting a diagram/graph, etc.

But when working with CAS we also have other modules which have a new quality. For example, we consider the process of solving equations: without CAS we have a module for solving quadratic equations, a module for solving linear equations, a module for solving polynomial equations, a module for solving trigonometric equations, etc. Using CAS the solve-module is one module for solving equations of all different types: solve(equation, var). Another example could be determining a derivative: without CAS we have different modules (rules) 


$$
\text { "Schneider" — 2009/2/18 — 23:40 — page 388 — \#10 }
$$

for deriving functions (product rule, quotient rule, chain rule,...). Using CAS we have one module for derivative, namely $d\left(\right.$ term, var) or $d(f(x), x)^{2}$.

Modules mostly represent experts' knowledge in compressed form. Using CAS-modules in an appropriate way demands from the students to explore and to investigate exactly those aspects of the modules which are also relevant for an appropriate communication with experts: the characteristics of the module, its conditions to use, its effects, its applicability, its range of validity, its limits,...

Therefore, the use of modules facilitates not only our thinking and acting, but it could also support reflection and the development of reflected knowledge about mathematical concepts.

Didactically, the idea of modularisation has not been used very often up to now - but in my opinion, it opens interesting possibilities.

\section{Using CAS is communication with experts}

Beside the contribution of CAS to the development of basic knowledge and reflection another useful aspect for general mathematics education focuses directly on the ability to communicate.

According to Fischer's theory of general mathematics education, we assign the field of competence for operative knowledge and skills primarily to experts. Mastering operational work is primarily knowledge of experts. Operative knowledge and skills lie in the same field of competence which could be delegated almost completely to CAS.

In this (narrow) sense we can perceive CAS as simple electronic models of mathematical experts. Of course, we can regard CAS as experts only in a limited way, because CAS have only limited basic knowledge of mathematics (which is almost restricted to operational knowledge and skills), limited abilities of representation and of interpretation; furthermore, CAS can sometimes even disappoint us in the operative field. Therefore, CAS can never become a substitute for human experts (and particularly not for teachers) - but in a way they can be seen as models for mathematical experts. Particularly, in using CAS, that is in the communication between human being and machine, many elements can be seen that are also quite significant for communication between laypersons and human experts: a successful and profitable interaction with human as well as electronic mathematical experts requires the following from the layperson:

${ }^{2} d \ldots$ derivative of 
- the willingness and ability to ask the "right" questions, to be precise when formulating one's own questions and to present them in a form which can be interpreted by the expert.

The communication with CAS assumes that the problem is selected in a way such that the question can really be answered by the expert - in our case CAS, i.e. that the expert CAS is really "competent" for the question. Then the problem has to be formulated precisely and with an adequate CAS-syntax. It is interesting that in the case of working with CAS a degree of exactness is required which even exceeds the exactness needed for communication with human experts. Inputs that have not been exactly understood by CAS inevitably lead to a non answered question or a "refusal to answer" in the way of an error message, demanding ("forcing") the user to be more precise (cf. Figure 6(a), 6(b)).

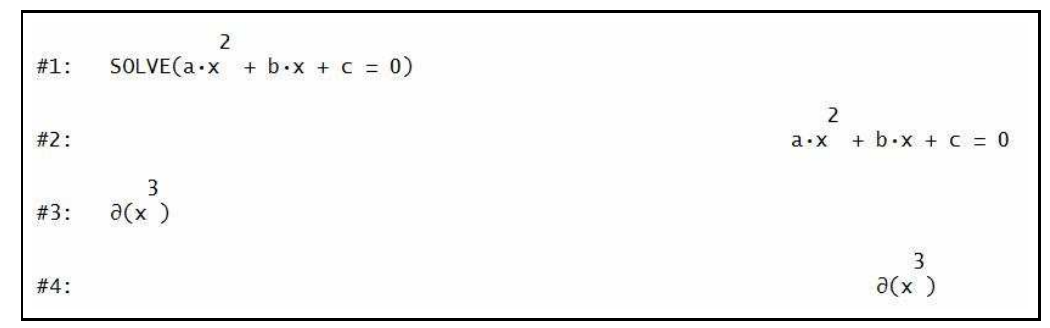

(a)

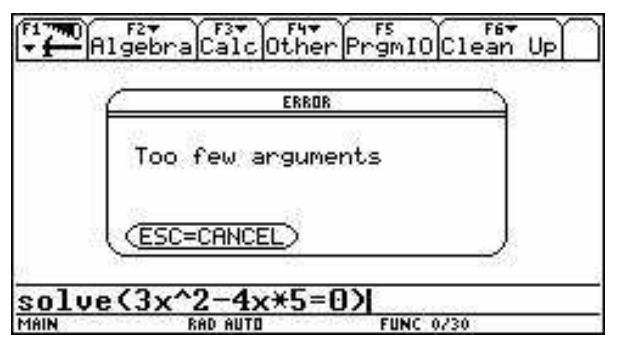

(b)

Figure 6

- exact conceptions of the possibilities and limits of the mathematical expert's knowledge.

The communication with CAS requires that one knows and is familiar with the prerequisites, effects, applicability, conditions of use and the limits of the 
modules offered by the expert CAS in order to guarantee an understanding and efficient use of "CAS-knowledge". For instance, it is necessary to know which equations or systems of equations could be solved by CAS-modules; in which of these cases one will be successful by using the solve-module, in which cases it would be better to solve the equations approximately by means of tables or graphs, etc.

- wide basic knowledge of mathematics.

Some examples:

It is very often necessary for the correct input of symbolic CAS-representations to be able to recognise the structure of the given formula and to be familiar with the hierarchy of arithmetical operations (cf. Figure 7).

$$
\frac{\frac{a^{2}}{a-2 \cdot b}+\frac{4 \cdot b^{2}}{2 \cdot b-a}-2 b}{\frac{\left(a^{\wedge} 2 /(a-2 b)\right)+\left(4 b^{\wedge} 2 /(2 b-a)>-2 b\right.}{\text { FAIN }}}
$$

Figure 7

Results of manipulations/transformations are sometimes given in unfamiliar formulas. This demands the ability to recognise the equivalence of expressions from the user, or at least to be acquainted with strategies how to verify the equivalence (cf. Figure 8(a), 8(b)).

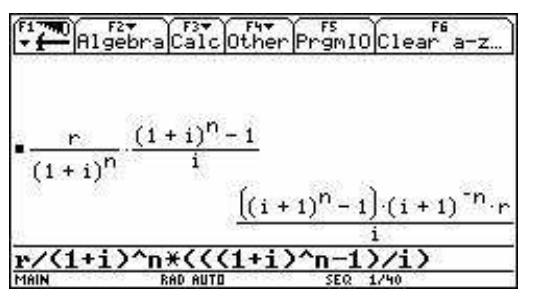

(a)

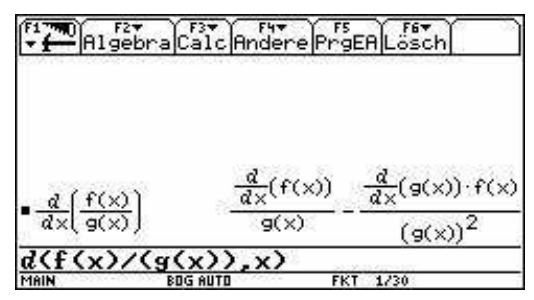

(b)

Figure 8

Basic knowledge is necessary for selecting an appropriate window (range of the screen) for a graphical representation. (Are all of the essential points of the graph visible on the screen?) It is also necessary for recognizing that 


$$
\text { "Schneider" — 2009/2/18 — 23:40 — page 391 — \#13 }
$$

the selected window is a relevant factor which determines the optical characteristics of the graph of a function (for instance, by zooming in the graph of an exponential function looks like a straight line), etc.

- a verification as well as an appropriate interpretation and assessment of the answers given by the expert.

The communication with CAS requires that the solutions shown by CAS must be recognised as the solution of an equation, the derivative of a function, etc. in order to be able to interpret them in context.

However, communication with CAS also requires an inner-mathematical verification, interpretation and evaluation of the solutions shown by CAS, such as: why does this equation have two solutions? Is that really possible? How can it be explained that the integral has a negative value? etc.

Regarding these elements, it can be useful to see and use CAS as simple models for the communication between mathematical experts and laypersons in mathematics classrooms. Reflection on CAS as such models for communication with experts can and also should be done with students. Using and reflecting CAS as such models could contribute to the development of an elaborated ability to communicate with mathematical experts.

Here, the development of appropriate teaching conceptions and materials appears to be a great and interesting challenge - results of investigations would be needed.

\section{Summary}

These considerations are an attempt to link two didactically challenging themes in an integrative way: general mathematics education (or mathematics education for everyone) on the one side and the use of Computer Algebra Systems (CAS) in the teaching of mathematics on the other side.

If one starts out with the ability to communicate with experts as the central aim of general mathematics education (mathematics education for everyone), the focus of teaching should lie in the development of competences in the fields of basic knowledge and reflection. The competences in the field of operative knowledge and skills are assigned primarily to the (mathematical) experts. At the same time, the operative knowledge and skills lie in that field of competence which could be mastered most completely by CAS. Hence, it could be outsourced to CAS. Therefore operational knowledge and skills are available for the students in the maths 


$$
\text { "Schneider" — 2009/2/18 — 23:40 — page 392 — \#14 }
$$

classrooms without the need for being developed by the students themselves. The availability of CAS in the classroom can be used as a pedagogical tool for the development of basic knowledge and reflection, namely by the possibilities of CAS in the fields of representation, exploration, elementarisation, and modularisation.

CAS also offer the possibility to take over the role of "simple" experts in the mathematics lessons. So CAS could be used and discussed as simple (electronic) models for communication with experts: CAS make "experts' knowledge" available; this experts' knowledge could become part of the communication in classrooms by asking for "experts' answers" as well as by justifying (assessing/evaluating) "experts' answers".

\section{References}

[1] Bildungsstandards im Fach Mathematik für den Mittleren Schulabschluss (Jahrgangsstufe 10), Kultusministerkonferenz, Luchterhand, 2003, http://www.kmk.org/schul/Bildungsstandards/Mathematik_MSA_BS_04-12-2003.pdf.

[2] W. Dörfler, Computer Use and Views of the Mind, in: Learning from Computers: Mathematics Education and Technology, (C. Keitel and K. Ruthven, eds.), Springer, Berlin - Heidelberg - New York, 1993, 159-181.

[3] R. Fischer, Höhere Allgemeinbildung und Bewusstsein der Gesellschaft, Erziehung und Unterricht 5-6 (2003), 559-566.

[4] R. Fischer, Höhere Allgemeinbildung, Typoskript, Universität Klagenfurt / Wien.

[5] W. Peschek, The Impact of CAS on our Understanding of Mathematics Education, The International Journal for Technology in Mathematics Education 14, no. 2 (2007), 95-101.

[6] W. Peschek and E. Schneider, How to identify basic knowledge and basic skills? Features of modern general education in Mathematics, International Journal of Computer Algebra in Mathematics Education 8, no. 1 (2001), 7-22.

[7] W. Peschek and E. Schneider, CAS in general mathematics education, Zentralblatt für Didaktik der Mathematik 5 (2002), 189-195.

[8] E. Schneider, Computeralgebrasysteme in einem allgemeinbildenden Mathematikunterricht, Didaktische Orientierungen - Praktische Erfahrungen, Profil, Wien München, 2002.

[9] E. Schneider, Kommunikation mit Expert/inn/en und Computeralgebrasysteme, in: Mathematik und Kommunikation, (S. Prediger, F. Siebel and K. Lengnink, eds.), Verlag Allgemeine Wissenschaft, Mühltal, 2002, 137-149.

[10] E. Schneider and W. Peschek, Computer Algebra Systems (CAS) and Mathematical Communication, International Journal of Computer Algebra in Mathematics Education 9, no. 3 (2002), 229-242. 


$$
\text { "Schneider" — 2009/2/18 — 23:40 — page 393 — \#15 }
$$

[11] Standards für die mathematischen Fähigkeiten österreichischer Schülerinnen und Schüler am Ende der 8. Schulstufe, Universität Klagenfurt, 2007, http://www.uni-klu.ac.at/idm/downloads/Standardkonzept_Version_4-07.pdf.

[12] H. J. Vollrath, Störungen des "didaktischen Gleichgewichts" im Mathematikunterricht, Der mathematische und naturwissenschaftliche Unterricht 40, no. 6 (1987), 373-378.

[13] H. Winter, Allgemeine Lernziele für den Mathematikunterricht, Zentralblatt für Didaktik der Mathematik 3 (1975), 106-116.

EDITH SCHNEIDER

INSTITUT FÜR DIDAKTIK DER MATHEMATIK

AUSTRIAN EDUCATIONAL COMPETENCE CENTRE FOR MATHEMATICS EDUCATION

ALPEN-ADRIA UNIVERSITÄT KLAGENFURT

STERNECKSTRASSE 15

9010 KLAGENFURT

AUSTRIA

E-mail: edith.schneider@uni-klu.ac.at

(Received September, 2007) 